\title{
Effect of Diane-35 on Polycystic Ovarian Syndrome of Different Subtypes
}

\author{
Yanqin Deng1*, Xiaoyan Deng2*\#, Haiyan Zhou², Yajun ${ }^{2 *}{ }^{2}$, Qing Luo², Jinli Li², Min Wang2, \\ Li Liu², Qing Xiao² \\ ${ }^{1}$ Chutian College Huazhong Agricultural University, Wuhan, China \\ ${ }^{2}$ Department of Gynecology and Obstetrics, The First Hospital of Wuhan, Wuhan, China \\ Email: \#xiaoyan6235@163.com
}

Received 22 June 2014; revised 18 July 2014; accepted 13 August 2014

Copyright (C) 2014 by authors and Scientific Research Publishing Inc.

This work is licensed under the Creative Commons Attribution International License (CC BY).

http://creativecommons.org/licenses/by/4.0/

(c) (i) Open Access

\begin{abstract}
Objective: To explore the effect of Daine-35 on serum hormone and antral follicle count of different subtypes of polycystic ovarian syndrome (PCOS). Methods: 138 cases of PCOS were recruited and typed according to Rotterdam diagnostic criteria; 78 cases of tubal infertility without hyperandrogenism and menstrual disorders during the same period were collected as control. Serum reproductive hormones including follicle-stimulating hormone (FSH), luteotropic hormone (LH), prolactin (PRL), estradiol (E2), dehydroepiandrosterone (DHEAS), total testosterone (TT) and fast insulin (INS) were determined by chemiluminescent immunoassay. Ovarian antral follicle count was determined by ultrasonography. The effects on above indexes were evaluated for different subtypes of PCOS after 3 cycles of treatment with Daine-35. Results: The cases of Type A and Type B were respectively $43(31.2 \%)$ and $73(52.9 \%)$. They were the most common clinical phenotypes of PCOS in the central region of China. Total testosterone (TT) and DHEAS levels of Types A, C and D were higher than those of the control group $(p<0.05)$. The LH, LH/FSH, INS and HOMA-IR levels in all types of PCOS were higher than those of the control group $(p<0.05)$. Antral follicle counts of Types A, B and D were more than those of Type $C$ and the control group $(p<0.05)$. After treatment with Daine-35 for 3 cycles, the serum TT levels of Types A, C and D decreased $(p<0.05)$. DHEAS values also decreased, but not significantly $(p>0.05)$. The serum LH and LH/FSH as well as the antral follicle count of all the PCOS types significantly decreased $(p<0.01)$. Conclusion: Type A and Type B are the most common clinical phenotypes of PCOS in the central region of China. Taking Daine-35 for 3 cycles can significantly reduce the serum androgen, serum LH, LH/FSH ratio, and antral follicle count of different PCOS types.
\end{abstract}

\section{Keywords}

Daine-35, Polycystic Ovarian Syndrome, Phenotype, Androgen, Antral Follicle Count

\footnotetext{
*These authors contributed equally to this work.

"Corresponding author.
}

How to cite this paper: Deng, Y.Q., Deng, X.Y., Zhou, H.Y., Hu, Y.J., Luo, Q., Li, J.L., Wang, M., Liu, L. and Xiao, Q. (2014) Effect of Diane-35 on Polycystic Ovarian Syndrome of Different Subtypes. Open Journal of Obstetrics and Gynecology, 4, 659-665. http://dx.doi.org/10.4236/ojog.2014.411092 


\section{Introduction}

Polycystic ovarian syndrome (PCOS) is a common ovulatory disorder in women, characterized by endocrine metabolic disorder and ovulatory dysfunction. The incidence of PCOS is approximately 4\% - 12\% [1] of women of childbearing age, and it is the main cause of anovulatory infertility (about 70\%). PCOS is of obvious heterogeneity; the commonly associated features such as hirsutism, acne, polycystic-appearing ovaries, obesity, and acanthosis nigricans are neither uniform nor universal. The diagnosis and typing criteria of PCOS have always been a contraversal issue [2]. By 1990 National Institutes of Health (NIH) meeting criteria, two principal phenotypes are generally recognized: women with anovulation and biochemical (or clinical) hyperandrogenism [3]. Most participants of this meeting advocated that the presence of polycystic ovaries by ultrasound (PCO-US) was suggestive, but not diagnostic for PCOS. Consequently, another expert conference was held in Rotterdam in 2003, recommending that PCOS could be defined when at least two of the following three features were present: 1) oligo- and/or anovulation (O/A); 2) hyperandrogenism (HA) or hirsutism [4]; 3) polycystic ovaries (PCO) and exclusion of other identifiable endocrine disorders such as late-onset congenital adrenal hyperplasia (CAH), hyperprolactinemia, thyroid dysfunction, neoplastic androgen secretion, or drug-induced androgen excess. Given this new PCOS definition, four clinical subtypes of PCOS cases become identifiable. ESHRE/ASRM criteria for PCOS diagnosis and typing proposed at annual conference in Rotterdam 2003 accord more with the demand of PCOS diagnosis for Chinese people [5] [6].

Daine-35 is commonly used to treat hyperandrogenism and menstrual disorders of PCOS. However, the effects of Daine-35 on different types of PCOS are still to be elucidated. To discuss this question, 138 cases of PCOS from the central region of China were collected and typed, according to the Rotterdam diagnostic criteria. These patients took Daine-35 orally for 3 cycles. Before and after treatment, serum endocrine hormone and ovarian antral follicle count of different PCOS types were analyzed.

The current study was undertaken to: 1 ) analyze the clinical, ultrasonographic (ovarian antral follicle count), hormonal and metabolic features of different type of PCOS; 2) compare the effects of Diane-35 on characteristics of different PCOS phenotype.

\section{Materials and Methods}

\subsection{Samples}

From December 2012 to March 2014, 138 cases of patients aged 15 - 39 (25.1 \pm 5.6$)$ and diagnosed according to the PCOS criteria proposed at Rotterdam conference in 2003 were collected [5]. The patients were all treated with Diane-35 and followed up. The patients were all treated with Diane-35 and followed up for three cycles (about three months). The participators were informed about the possible side effects of this drug. As a control group, another 78 cases of tubal infertility younger than 40 (26.2 \pm 5.4$)$, with no hyperandrogenism and menstrual disorders, were selected at the same period. Informed consent was obtained from all the participators before they were enrolled in this study, and this investigation was approved by the Ethics Committee of the First Hospital of Wuhan.

\subsection{PCOS Criteria and Classification}

The disease meeting any two or all of the following three conditions were diagnosed as PCOS: 1) oligo- and/or anovulation (O/A); 2) hyperandrogenism (HA) or hirsutism [4]; 3) polycystic ovaries (PCO). The patients with other disorders (such as 21-hydroxylase deficiency, Cushion's syndrome, thyroid dysfunction, cancer and endocrine function with premature ovarian failure, etc.), which can also lead to increased androgen or anovulation, or patients aged older than 40 years, or with FSH $>25 \mathrm{IU} / \mathrm{L}, \mathrm{PRL}>24 \mu \mathrm{g} / \mathrm{L}$, thyroid stimulating hormone (TSH) > $5.5 \mathrm{mIU} / \mathrm{L}$, hyperlipidemia, and other acute or chronic diseases were excluded.

In detail, oligoovulation or anovulation was determined when the menstrual cycle is less than 23 days or more than 35 days, and no evidence of ovulation was test; hirsutism refers to F-G (Ferriman-Gallwey) score valuing greater than or equal to 5 [7]. Polycystic ovaries was determined according to the ovarian volume or follicles number, using transvaginal and transrectal ultrasound, with unilateral ovarian volume greater than $10 \mathrm{ml}$, or at least one ovary containing more than 12 small follicles (2 - $9 \mathrm{~mm}$ in diameter). Homeostasis model of assessment for insulin resistance (HOMA-IR) was calculated according to the formula INS (mIU/L) $\times$ FBG $(\mathrm{mmol} / \mathrm{L}) / 22.5$. According to the results based on large sample of Chinese population study, HOMA-IR valuing greater than or 
equal to 1.66 was determined as insulin resistance [2]. Based on the above different conditions, the 138 patients was divided into 4 types: Type A (O/A + HA + PCO), with oligoovulation or anovulation, hyperandrogenism and polycystic ovaries; Type B (O/A + PCO), oligoovulation or no ovulation and polycystic ovaries; Type C (O/A + HA), oligoovulation or anovulation, increased androgen levels (clinical and (or) biochemical analysis); Type D (HA + PCO), menstrual cycle being normal, with hyperandrogenism and polycystic ovaries.

\subsection{Serum Endocrine Hormone and Ovarian Detection}

During the 3rd - 5th days of menstruation or any day for patients with amenorrhea, fasting blood samples were collected. Using chemiluminescence immunoassay, the following serum endocrine hormone were measured, including luteinizing hormone (LH), follicle stimulating hormone (FSH), estradiol (E2), total testosterone (TT), prolactin (PRL), dehydroepiandrosterone sulfate (DHEAS) and fasting insulin (INS). For patients with amenorrhea, progesterone was measured to determine the condition of ovulation or anovulation. Dry chemistry method was used to determine fasting blood glucose (FBG). Total plasma cholesterol and triglyceride was also measured. Ovaries of three warp $\left(\mathrm{d}_{1}, \mathrm{~d}_{2}, \mathrm{~d}_{3}\right)$ was measured by transvaginal or transrectal ultrasound, using Color Doppler ultrasonography (TOSHIBA-585G, $5 \mathrm{MHz}$ ), and ovarian volume was calculated [the formula 4/3 $\pi$ ( $\mathrm{d}_{1}$ $\times d_{2} \times d_{3}$ )]. Antral follicles count (AFC) was measured as the total number of follicles at both sides.

\subsection{Diane-35 Treatment}

From the 5th day of menstrual cycle or any day for patients with amenorrhea, Diane-35 (Schering AG, Germany production Zhunzi J20100003) was taken orally, once per day, lasting for 21 days. This therapy treatment was continued for three menstrual cycles. After that, at the 3rd day of menstrual cycle, the serum endocrine hormone and ovarian were detected as detailed above. Considering adverse effects of Diane-35 on blood lipids, patients with a history of thrombosis, cancer and liver disease or with high levels of blood cholesterol were excluded in this study. During the treatment period, the patients were required to visit the doctors if they feel any discomfort. In this study, except mild nausea and lethargy, no other side effects occurred.

\subsection{Statistical Methods}

Levene analysis of variance and T-test, as well as the chi-square test were measurement (SPSS 19.0). Test level $\alpha=0.05, \mathrm{p}<0.05$ was considered statistically significant.

\section{Results}

\subsection{The Patients and PCOS Classification}

Among the 138 patients with PCOS, 131 cases did not ovulate or be oligomenorrhea, accounting for 94.9\%. PCO occurred in 123 cases, accounting for 89.1\% of all patients. Hyperandrogenism occurred in 46 cases, accounting for 33.3\%. 19 patients were with hirsutism, accounting for $13.8 \%$. Patients with hyperandrogenism or hirsutism were total 65 cases, accounting for $47.1 \%$. 47 cases were obese, accounting for 34.1\%. In the control group, 24 cases were obese (30.8\%), whose weight was not significantly different from the PCOS group. The plasma total cholesterol and triglyceride levels of all participants were within the normal range. PRL and E2 level of every subtypes were also within the normal range and not significantly different from that of the control group (Table 1 ).

As displayed in Table 1, the most common type among the four PCOS types is Type B (73 cases, 52.9\%) in this study, followed by Type A (43 cases, 31.2\%). Type C (15 cases, 10.8\%) followed after Type A, and have no symptoms of PCO or enlarged ovaries. Type D is the most rare (7 cases, 5.1\%), and patients of this type have no clinical manifestations of menstrual disorders or anovulation. Some of these patients were diagnosed because of infertility or miscarriage, and some were also because of skin problems (hirsutism, acne, etc.), and be transferred to our department of gynaecology. Age and BMI of various PCOS types were not significantly different from the control group. F-G values show some differences among the four types (Table 1). In detail, types of A, C, and D have significant ( $\mathrm{p}<0.05$ ) higher F-G score compared to the control group. F-G score of Type B were higher than that of the control group but not significantly $(p>0.05)$. 
Table 1. Distributions and clinical characteristics of various PCOS types.

\begin{tabular}{ccccccc}
\hline & Cases (\%) & Age (years) & BMI $\left(\mathrm{kg} / \mathrm{m}^{2}\right)$ & F-G score & PRL (mIU/L) & E2 (pmol/L) \\
\hline Type A & $43(31.2 \%)$ & $24.9 \pm 5.2$ & $22.1 \pm 4.9$ & $5.8 \pm 4.1^{\mathrm{a}}$ & $262.3 \pm 106.6$ & $143.7 \pm 55.4$ \\
Type B & $73(52.9 \%)$ & $25.7 \pm 5.4$ & $23.6 \pm 5.1$ & $2.8 \pm 1.6$ & $220.6 \pm 89.7$ & $160.6 \pm 68.0$ \\
Type C & $15(10.8 \%)$ & $25.3 \pm 4.9$ & $23.2 \pm 5.9$ & $4.3 \pm 3.0^{\mathrm{a}}$ & $300.6 \pm 117.7$ & $152.2 \pm 59.4$ \\
Type D & $7(5.1 \%)$ & $24.4 \pm 5.5$ & $22.8 \pm 4.2$ & $3.4 \pm 1.5^{\mathrm{a}}$ & $255.6 \pm 92.0$ & $187.6 \pm 85.9$ \\
Control & 78 & $26.2 \pm 5.4$ & $22.2 \pm 2.4$ & $2.2 \pm 1.2$ & $286.2 \pm 86.4$ & $147.2 \pm 32.5$ \\
\hline
\end{tabular}

Denotes: ${ }^{\mathrm{a} C o m p a r e d ~ t o ~ t h e ~ c o n t r o l ~ g r o u p, ~} \mathrm{p}<0.05$.

\subsection{Serum Endocrine Hormone and Ovarian Conditions of the Various PCOS Types}

Compared to the control group, TT and DHEAS levels of Type A, C and D of PCOS patients were significantly higher ( $p<0.05$, Table 2, pre-treatment). TT levels of Type B were higher than the control group, but not significantly ( $p>0.05$ ). LH and LH/FSH ratio of various types of PCOS patients were significantly higher than the control ( $<<0.05$ ). Compared to the control group, fasting blood glucose levels of various types of PCOS patients was not statistically significant $(\mathrm{p}>0.05)$. However, fasting insulin and HOMA-IR values of these patients were significantly higher than the control group $(\mathrm{p}<0.05)$. The antral follicles of Type A, B and D count more than the Type $C$ and the control group $(p<0.05)$. Although antral follicles of Type $C$ count more than the control group and that of Type A count more than Type B, the difference was not significant ( $p>0.05$ ).

\subsection{Serum Endocrine Hormone and Ovarian Conditions Changes after Diane-35 Treatment}

As shown in Table 2 (bottom row), after Diane-35 therapy treatment for three cycles, serum LH levels and LH/FSH values of the various types (A, B, C and D) of PCOS patients significantly decreased ( $<0.05)$. Serum TT levels of Type A, C and D of PCOS patients were significantly reduced $(\mathrm{p}<0.05)$. DHEAS levels of these three types were also reduced, but not significantly $(\mathrm{p}>0.05)$. The antral follicle numbers of Type A, B and D of PCOS patients significantly decreased $(\mathrm{p}<0.05)$. After Diane-35 treatment, fasting glucose, fasting insulin and HOMA-IR of various PCOS types increased, but not significantly ( $>>0.05)$. No obvious change of F-G score of the PCOS patients was observed.

\section{Discussions}

Previous researches about the distributions of different PCOS types were different from each other [8]-[10], possibly due to different region, ethnicity and diagnostic criteria. In this study, Type A with oligo- and/or anovulation, hyperandrogenism, and polycystic ovary revealed by ultrasonography (O/A + HA + PCO) accounted for $31.2 \%$. It is a classic type with serious endocrine and metabolic disorders [11] [12]. Type B with oligoand/or anovulation and polycystic ovary (O/A + PCO) accounted for 52.9\%. Although the PCOS diagnostic criteria of USA National Institutes of Health (NIH) in 1990 and of Androgen Excess Association (AES) in 2006 have both excluded it, Type B is one of the most common types of Chinese patients [13]. Type C accounted for $10.8 \%$, with clinical and/or biochemical signs of hyperandrogenism, and oligo- and/or anovulation (HA + O/A). This type and classic Type A PCOS patients have similar clinical manifestations and metabolism level. However, as patients of this type have less antral follicles than Type A, it needs to be further studied if Type C patients are more sensitive to the ovulation drugs than Type A patients. The proportion of Type D patients is $5.1 \%$, with no menstrual disorders and ovulation disorders. They are often diagnosed firstly because of infertility or reduplicated abortion, even of the skin manifestations (hirsutism, acne and so on). The actual proportion may be higher than the clinical statistics because of sub-clinical manifestations. Total proportion of Type B and D were 58.0\%, which met the diagnostic criteria of ESHRE/ASRM 2003, but not that of NIH 1990. Our results were consistent with the study of Zhang et al. [2], which included a large-scale sample of 719 Chinese PCOS women who were all from the Han nationality and were diagnosed based on the Rotterdam criteria of 2003. They found that 247 cases (34.4\%) of the PCOS patients met both the diagnostic criteria of NIH 1990 and ESHRE/ASRM 2003, and 
Table 2. Comparison of serum endocrine hormone levels and ovarian AFC of different types of PCOS patients before (upper row) and after (under row) Diane-35 treatment (mean $\pm \mathrm{SD}$ ).

\begin{tabular}{|c|c|c|c|c|c|}
\hline & Type A $(n=43)$ & Type B $(\mathrm{n}=73)$ & Type C $(n=15)$ & Type D $(\mathrm{n}=7)$ & Control $(\mathrm{n}=78)$ \\
\hline \multirow[t]{2}{*}{ LH (IU/L) } & $9.2 \pm 6.0^{\mathrm{a}}$ & $9.4 \pm 5.5^{\mathrm{a}}$ & $9.1 \pm 4.5^{\mathrm{a}}$ & $6.1 \pm 3.3^{\mathrm{a}}$ & $3.1 \pm 1.2$ \\
\hline & $4.5 \pm 2.1^{\mathrm{c}}$ & $5.0 \pm 1.8^{\mathrm{c}}$ & $5.2 \pm 3.8^{c}$ & $4.0 \pm 2.8^{c}$ & \\
\hline \multirow[t]{2}{*}{ LH/FSH } & $2.3 \pm 2.2^{\mathrm{a}}$ & $1.7 \pm 0.9^{\mathrm{a}}$ & $1.9 \pm 1.0^{\mathrm{a}}$ & $1.5 \pm 0.3^{\mathrm{a}}$ & $0.6 \pm 0.2$ \\
\hline & $1.1 \pm 0.4^{\mathrm{c}}$ & $0.9 \pm 0.3^{c}$ & $1.1 \pm 0.5^{c}$ & $0.8 \pm 0.2^{c}$ & \\
\hline \multirow[t]{2}{*}{$\mathrm{TT}(\mathrm{nmol} / \mathrm{L})$} & $2.8 \pm 1.4^{\mathrm{a}, \mathrm{b}}$ & $1.8 \pm 1.1$ & $2.9 \pm 1.7^{\mathrm{a}, \mathrm{b}}$ & $2.6 \pm 1.1^{\mathrm{a}, \mathrm{b}}$ & $1.3 \pm 0.5$ \\
\hline & $1.6 \pm 1.1^{\mathrm{c}}$ & $1.5 \pm 1.3$ & $1.8 \pm 1.5^{c}$ & $1.5 \pm 1.3^{c}$ & \\
\hline \multirow[t]{2}{*}{ DHEAS $(\mu \mathrm{mol} / \mathrm{L})$} & $6.7 \pm 4.3^{\mathrm{a}}$ & $5.4 \pm 2.8$ & $6.8 \pm 3.7^{\mathrm{a}}$ & $7.1 \pm 4.0^{\mathrm{a}}$ & $4.1 \pm 1.4$ \\
\hline & $5.9 \pm 3.3$ & $4.9 \pm 3.7$ & $6.2 \pm 3.5$ & $5.8 \pm 2.3$ & \\
\hline \multirow[t]{2}{*}{ FBG $(\mathrm{mmol} / \mathrm{L})$} & $4.5 \pm 0.7$ & $5.4 \pm 2.0$ & $4.7 \pm 1.1$ & $4.2 \pm 0.8$ & $4.4 \pm 0.4$ \\
\hline & $4.3 \pm 1.9$ & $5.5 \pm 1.7$ & $4.5 \pm 1.0$ & $5.1 \pm 1.4$ & \\
\hline \multirow[t]{2}{*}{ INS (mIU/L) } & $11.3 \pm 4.7^{\mathrm{a}}$ & $10.3 \pm 5.8^{\mathrm{a}}$ & $9.5 \pm 3.5^{\mathrm{a}}$ & $12.0 \pm 5.1^{\mathrm{a}}$ & $6.0 \pm 2.7$ \\
\hline & $12.5 \pm 5.5$ & $11.0 \pm 4.6$ & $10.8 \pm 4.2$ & $13.1 \pm 4.8$ & \\
\hline \multirow[t]{2}{*}{ HOMA-IR } & $2.3 \pm 1.4^{\mathrm{a}}$ & $2.5 \pm 1.8^{\mathrm{a}}$ & $2.0 \pm 1.4^{\mathrm{a}}$ & $2.2 \pm 1.5^{\mathrm{a}}$ & $1.1 \pm 0.7$ \\
\hline & $2.4 \pm 1.3$ & $2.7 \pm 1.7$ & $2.2 \pm 1.5$ & $2.9 \pm 2.1$ & \\
\hline \multirow[t]{2}{*}{ AFC (No.) } & $28.3 \pm 8.9^{\mathrm{a}}$ & $25.7 \pm 6.0^{\mathrm{a}}$ & $18.3 \pm 4.5$ & $24.6 \pm 3.9^{\mathrm{a}}$ & $15.2 \pm 4.2$ \\
\hline & $19.8 \pm 4.8^{\mathrm{c}}$ & $18.4 \pm 3.9^{c}$ & $16.2 \pm 3.4$ & $17.3 \pm 5.7^{\mathrm{c}}$ & \\
\hline
\end{tabular}

Denote: ${ }^{\mathrm{a}}$ In comparison to control group, $\mathrm{p}<0.05$; ${ }^{\mathrm{b}}$ In comparison to Type B, $\mathrm{p}<0.05$; ${ }^{\mathrm{c}}$ In comparison to that of PCOS patients before Diane-35 treatment, $\mathrm{p}<0.05$.

the proportion of new phenotypes created by the ESHRE/ASRM 2003 was 65.6\%. As shown in Table 1, Type B accounts for a large proportion of Chinese PCOS, who are often urgent to visit a doctor for menstrual disorder or infertility, too. Therefore, the Rotterdam ESHRE/ASRM criteria for the diagnosis of PCOS is more suitable for Chinese women.

Compared to the control group, serum LH, TT levels and LH/FSH ratio of PCOS patients were all increased (Table 2). That means regulation disorder of sex hormone in PCOS patients. It's well known that the serum levels of TT and DHEAS are good indicators of hyperandrogenism, and elevated androgen levels and insulin resistance are basic features of PCOS. Therefore, elevated levels of LH and LH/FSH ratio may be the results of increasing serum androgen and insulin resistance. There was no difference of fasting blood glucose between the various types of PCOS and the control. That may be owing to their young age and matching body mass index to the control group (Table 1). Fasting insulin (INS) and HOMA-IR were all higher in various types of PCOS than those in the control group (Table 2). The results suggested that insulin resistance occurred even at the early stage in young PCOS patients. So it is important to detect insulin resistance of PCOS patients for monitor and treatment of metabolic syndrome as early as possible.

Recent researches have revealed that PCOS should be viewed as not only a syndrome affecting reproduction but also a multifaceted endocrine syndrome that potentially threatens health. For example, cardiovascular disease, endodermis dysfunction, and abnormal lipometabolism were all correlated with this syndrome [14]. In 2011, guidelines issued by China's Ministry of Health for PCOS pointed out that the classic PCOS patients (abnormal menstruation and hyperandrogenism, with or without PCO, just as Type A and C patients in this study) have serious metabolic disorders. On the other hand, those PCOS patients without hyperandrogenism (only the abnormal menstruation and PCO, just as Type B patients in this study) have slight metabolic disorder. In this study, taking into account the fact that Daine-35 may have adverse effects on blood lipids levels [12], those patients with elevated blood lipids had been excluded. Plasma total cholesterol and triglyceride levels of all participants were within the normal range. Androgen levels and LH/FSH ratio were significantly higher in patients of 
Type A and C than those of Type B (Table 2). The results suggested that the endocrine disorders of Type A and $\mathrm{C}$ are more serious than Type B. No obvious difference of insulin homeostasis assessment index (HOMA-IR) was observed. Differences in metabolic disorders and complications in patients of different type need further study by increasing the sample size.

There was a significant difference of serum androgen level and clinical manifestations of excessive androgen among different race [15]. Although neither meeting to the $1990 \mathrm{NIH}$ nor to 2006 AES diagnostic criteria, Type B PCOS are mainly involved patients in the clinic of China for menstrual disorders, anovulation and infertility. This study showed that TT levels of Type B were in normal range, but still higher than that of the control (Table 2). It indicated that androgen may be also involved in the pathogenesis in Type B PCOS. The serum androgen level and mechanism of Type B PCOS in Chinese people need further research. Type C and Type D PCOS are in low incidence in China. Their interaction with Type A and Type B remains to be further studied. It is indicated that PCOS without polycystic ovary (that is Type $\mathrm{C}$ in our observations) represents a new subgroup of PCOS with higher total cholesterol, LDL and F-G grade than in patients with classic polycystic ovary [16]. It needs observations of more samples to support that conclusion.

Daine-35 is effective in correcting endocrine disorders, regulating menstrual cycle, reducing the risk of endometrial cancer of PCOS patients [17]. To our knowledge, researches about the efficacy of Daine-35 for different types of PCOS were rare. Daine-35 is a compound tablet combined of small dose of cyproterone acetate and ethinyl estradiol. It can inhibit the hypothalamic pituitary ovarian axis effectively and reduce ovarian androgen secretion. At the same time it promotes the synthesis of sex hormone binding globulin and then the free testosterone level decreases. It also reportedly inhibits the androgen secretion of adrenal [18]. We pay close attention to controlling $\mathrm{T}$ levels in the management of PCOS patients, because $\mathrm{T}$ underpins all other manifestations of the syndrome. In this study, LH, LH/FSH of each type of PCOS decreased after Daine-35 treatments for three cycles. Total testosterone level and antral follicle count also significantly reduced (Table 2). It indicated that Daine-35 have played a positive role in adjusting the endocrine disorders of various type of PCOS. Although the F-G scores did not change significantly in this research after treatment for 3 cycles, Daine-35 is an effective treatment medicine for Hirsutism in PCOS, but the course of treatment need to be above 6 cycles [19].

\section{Conclusion}

Previous work has shown that the risk for adverse obstetric or neonatal outcomes was affected significantly by ovarian dysfunction and biochemical hyperandrogenism, whereas no significant effect was detected for clinical hyperandrogenism and PCO [20]. Therefore, to those PCOS patients who have a history of abortion, it is speculated that controlling serum androgen levels by Daine-35 would be conducive to improve the pregnancy rate and reduce the abortion rate. There are two limitations in this study, one is a small size of sample (especially D group), and the other is relatively short follow-up time (3 cycles only). To resolve the above problems, larger sample size and longer term effects of Daine-35 on serum hormone of different types of PCOS and effects on pregnant outcome need to be investigated further.

\section{Funding}

This project is supported by fund of Wuhan Science and Technology Bureau (2013062301010815).

\section{References}

[1] Asuncion, M., Calvo, R.M., San Millan, J.L., et al. (2000) A Prospective Study of the Prevalence of the Polycystic Ovary Syndrome in Unselected Caucasian Women from Spain. The Journal of Clinical Endocrinology \& Metabolism, 85, 2434.

[2] Zhang, H.Y., Guo, C.X., Zhu, F.F., et al. (2013) Clinical Characteristics, Metabolic Features, and Phenotype of Chinese Women with Polycystic Ovary Syndrome: A Large-Scale Case-Control Study. Archives of Gynecology and Obstetrics, 287, 525-531. http://dx.doi.org/10.1007/s00404-012-2568-z

[3] Zawadski, J.K. and Dunaif, A. (1992) Diagnostic Criteria for Polycystic Ovary Syndrome: Towards a Rational Approach. In: Dunaif, A., Givens, J.R. and Haseltine, F., Eds., Polycystic Ovary Syndrome, Blackwell Scientific, Boston, 377-384.

[4] Du, S.M., Ma, M.J., Lin, D.Y., et al. (2013) Consensus on the Women's Health of PCOS: The Amsterdam ESHRE/ ASRM-Sponsored 3rd PCOS Consensus Workshop Group. Journal of International Reproductive Health/Family 
Planning, 32, 222-225.

[5] The Rotterdam ESHRE/ASRM-Sponsored PCOS Consensus Workshop Group (2004) Revised 2003 Consensus on Diagnostic Criteria and Long-Term Health Risks Related to Polycystic Ovary Syndrome. Fertility and Sterility, 81, 19-25.

[6] Zhang, S.D., Zhang, H.L., Li, M., et al. (2014) Compared the Clinical and Biochemical Features of Different Phenotypic Subtype of Polycystic Ovary Syndrome. Journal of Reproductive Medicine, 23, 100-104.

[7] Zhao, X., Ni, R., Li, L., et al. (2011) Defining Hirsutism in Chinese Women: A Cross-Sectional Study. Fertility and Sterility, 96, 792-796. http://dx.doi.org/10.1016/j.fertnstert.2011.06.040

[8] Baldani, D.P., Skrgatic, L., et al. (2013) Characteristics of Different Phenotypes of Polycystic Ovary Syndrome Based on the Rotterdam Criteria in the Croatian Population. Collegium Antropologicum, 37, 477-482.

[9] Hong, Y.Z., Cheng, X.G., Fu, F.Z., et al. (2013) Clinical Characteristics, Metabolic Features, and Phenotype of Chinese Women with Polycystic Ovary Syndrome: A Large-Scale Case-Control Study. Archives of Gynecology and Obstetrics, 287, 525-531. http://dx.doi.org/10.1007/s00404-012-2568-Z

[10] Zhao, Y., Fu, L., Li, R., Wang, L.N., Yang, Y., Liu, N.N., et al. (2012) Metabolic Profiles Characterizing Different Phenotypes of Polycystic Ovary Syndrome: Plasma Metabolomics Analysis. BMC Medicine, 10, 153. http://dx.doi.org/10.1186/1741-7015-10-153

[11] Cui, L.L. and Chen, Z.J. (2011) Diagnosis Criteria and Guidelines for the Diagnosis and Treatment of PCOS. Journal of International Reproductive Health/Family Planning, 30, 405-408.

[12] Lin J.F., Li, X. and Zhu, M.W. (2006) Exploration of the Classification of Polycystic Ovarian Syndrome. Chinese Journal of Obstetrics and Gynecology, 41, 684-688.

[13] Li, M., Song, J., Chen, S.L., Luo, C., Ni, Y.P., Wang, Q.L., et al. (2009) Association of the Clinical Characteristics and the IVF-ET Outcome in Infertile Women with Polycystic Ovarian Syndrome of Different Subtypes. Journal of South Medicine University, 29, 224-227.

[14] Orio, F., Cascella, T., Giallauria, F., Palomba, S., De Lorenzo, A., Lucci, R., et al. (2006) Polycystic Ovary Syndrome: An Example of Obesity-Related Cardiovascular Complication Affecting Young Women. Monadi Archives for Chest Disease, 66, 48-53.

[15] Zhao, Y. and Qiao, J. (2013) Ethnic Differences in the Phenotypic Expression of Polycystic Ovary Syndrome. Steroids, 78, 755-760. http://dx.doi.org/10.1016/j.steroids.2013.04.006

[16] Shi, Y., Gao, X., Sun, X., Zhang, P. and Chen, Z. (2008) Clinical and Metabolic Characteristics of Polycystic Ovary Syndrome without Polycystic Ovary: A Pilot Study on Chinese Women. Fertility and Sterility, 90, 1139-1143. http://dx.doi.org/10.1016/j.fertnstert.2007.07.1375

[17] Li, X., Guo, Y.R., Lin, J.F., Feng, Y., Billig, H. and Shao, R. (2014) Combination of Diane-35 and Metformin to Treat Early Endometrial Carcinoma in PCOS Women with Insulin Resistance. Journal of Cancer, 5, 173-181. http://dx.doi.org/10.7150/jca.8009

[18] Zhang, X.Y. (2003) The Utility of Obstetrics and Gynecology. 2nd Edition, 829-831.

[19] Tartagni, M., Schonauer, L.M., De Salvia, M.A., Cicinelli, E., De Pergola, G. and D’Addario, V. (2000) Comparison of Diane 35 and Diane 35 plus Finasteride in the Treatment of Hirsutism. Fertility and Sterility, 73, 718-723. http://dx.doi.org/10.1016/S0015-0282(99)00633-0

[20] Palomba, S., Falbo, A., Russo, T., Tolino, A., Orio, F. and Zullo, F. (2010) Pregnancy in Women with Polycystic Ovary Syndrome: The Effect of Different Phenotypes and Features on Obstetric and Neonatal Outcomes. Fertility and Sterility, 94, 1805-1811. http://dx.doi.org/10.1016/j.fertnstert.2009.10.043 
Scientific Research Publishing (SCIRP) is one of the largest Open Access journal publishers. It is currently publishing more than 200 open access, online, peer-reviewed journals covering a wide range of academic disciplines. SCIRP serves the worldwide academic communities and contributes to the progress and application of science with its publication.

Other selected journals from SCIRP are listed as below. Submit your manuscript to us via either submit@scirp.org or Online Submission Portal.
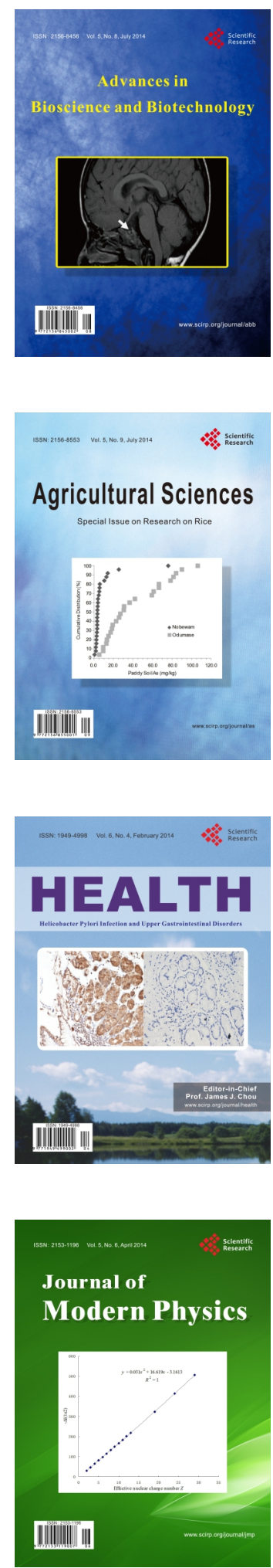
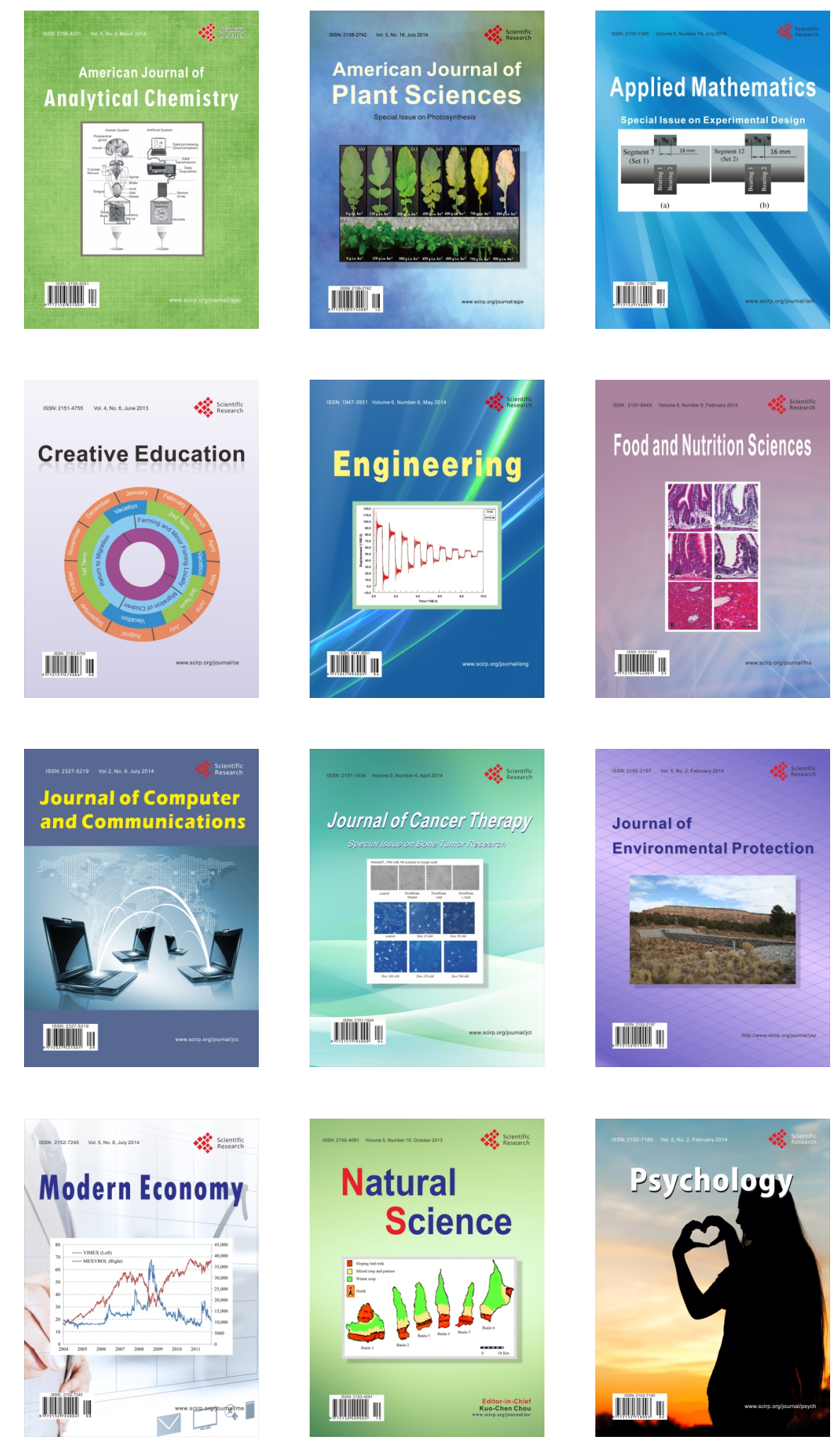